\title{
Bioinspired tuning of glycol chitosan for 3D cell culture
}

\author{
Myeong Ok Cho ${ }^{1,2,7}$, Zhengzheng $\mathrm{Li}^{1,3,7}$, Hye-Eun Shim ${ }^{2,7}$, Ik-Sung Cho ${ }^{1}$, Md Nurunnabi ${ }^{1}$, Honghyun Park ${ }^{4}$, \\ Kuen Yong Lee ${ }^{4}$, Sung-Hwan Moon ${ }^{5}$, Ki-Suk Kim² ${ }^{2}$, Sun-Woong Kang ${ }^{2,6,8}$ and Kang Moo Huh ${ }^{1,8}$
}

Three-dimensional (3D) cell culture systems have promising applications compared with conventional two-dimensional cell culture systems. Herein, we report a facile method for the formation of 3D spheroids using novel thermo-reversible polysaccharide-based hydrogels. A series of thermo-reversible hydrogels consisting of $\mathrm{N}$-acyl glycol chitosans (NAGCs) are synthesized through a simple $\mathrm{N}$-acylation reaction, and the degree of acylation is finely tuned to obtain adequate thermoreversible properties and gel stability. Among the NAGCs, $\mathbf{N}$-hexanoyl glycol chitosan is the most thermo-sensitive and is highly effective for forming multi-cellular spheroids when used to coat the surfaces of cell culture dishes. Cell spheroids are effectively formed at various cell concentrations, and their spheroid shape and cellular functions are well maintained for longer times. The hydrogel culture system is also useful for co-cultures that mimic a biological microenvironment. Our thermo-reversible hydrogels may offer a convenient method for the development of in vitro 3D cell culture systems to provide enhanced performance in tissue regeneration, organ-on-chips, drug screening research and other biomedical applications.

NPG Asia Materials (2016) 8, e309; doi:10.1038/am.2016.130; published online 9 September 2016

\section{INTRODUCTION}

Three-dimensional (3D) spheroid culture is an important process for understanding biological mechanisms and obtaining transplantable cells for therapy. Spheroid culture systems present a biomimetic environment that can provide a cellular microenvironment with significant effects on surrounding cells in terms of structural and functional properties. ${ }^{1}$ Several types of spheroid culture methods, such as the hanging drop method, micro-molding, centrifugation and the use of multi-well hanging drop plates, have been utilized to support in vivo-like $3 \mathrm{D}$ cellular environment and mimicking physiological tissues. ${ }^{2-5}$ However, these systems often require sophisticated microdevices, limit mass production, impede long culture durations and require the use of inconvenient processes to retrieve spheroids for further analysis. ${ }^{6}$

An ideal method for spheroid formation must be convenient, allow for mass production, and allow cells to be cultivable for long periods of time and easily retrievable. Recently, non-cell adherent polymers have been utilized for $3 \mathrm{D}$ spheroid formation and culture. ${ }^{7-9}$ In this case, cells may not adhere to the substrate but could form spheroids on the non-adherent substrate because cell-cell interactions are favored over cell-substrate interactions. In this regard, chitosan, a deacetylated chitin, has been widely studied as a non-adherent polymer to promote the formation of self-assembled 3D cellular spheroids. ${ }^{10-12}$ However, chitosan is soluble only in an acidic environment, which may be potentially cytotoxic to cells. Therefore, this method requires an extensive washing process to remove cytotoxic conditions before the addition of cells for spheroid formation. In addition, in most cases, the conservation of the spheroids that are formed is problematic, most likely due to re-adhesion on the plate $\mathrm{e}^{10-12}$, which limits the prolonged culturing and retrievability of the $3 \mathrm{D}$ spheroids. Thus, the non-cell adherent property of chitosan itself seems insufficient for successful 3D spheroid culture.

The design and synthesis of innovative chitosan derivatives is still a major goal for effective 3D spheroid formation and culture. Numerous chemical modification methods allow for the requirements of specific applications to be met. ${ }^{13}$ To develop a versatile, alternative method for producing and maintaining a variety of spheroids with different cell types, it is critical for chitosan to have adequate physico-chemical properties and enhanced non-adhesive properties imparted by chemical modification. Temperature-tunable physical properties that undergo a thermo-responsive sol-gel transition enable harvesting of cells without enzymatic digestion, such as trypsinization, which results in the degradation of cell surface proteins. In addition, the temperature-tunable physical properties may facilitate the preparation

\footnotetext{
${ }^{1}$ Department of Polymer Science and Engineering, Chungnam National University, Daejeon, Republic of Korea; ${ }^{2}$ Next-generation Pharmaceutical Research Center, Korea Institute of Toxicology, Daejeon, Republic of Korea; ${ }^{3}$ School of Chemical Engineering and Materials, Tianjin University of Science \& Technology, Tianjin, China; ${ }^{4}$ Department of Bioengineering, Hanyang University, Seoul, Republic of Korea; ${ }^{5}$ Department of Medicine, School of Medicine, Konkuk University, Seoul, Republic of Korea and ${ }^{6}$ Department of Human and Environmental Toxicology, University of Science and Technology, Daejeon, Republic of Korea

${ }^{7}$ These authors contributed equally to this paper as first authors.

${ }^{8}$ These authors contributed equally to this paper as corresponding authors.

Correspondence: Dr S-W Kang, Next-generation Pharmaceutical Research Center, Korea Institute of Toxicology, 141 Gajeong-ro, Yuseong-gu, Daejeon 305-343, Republic of Korea.

E-mail: swkang@kitox.re.kr

or Professor KM Huh, Department of Polymer Science and Engineering, Chungnam National University, Daejeon 305-764, Republic of Korea.

E-mail: khuh@cnu.ac.kr

Received 19 March 2016; revised 25 June 2016; accepted 6 July 2016
} 


\section{Thermogelation of HGC}
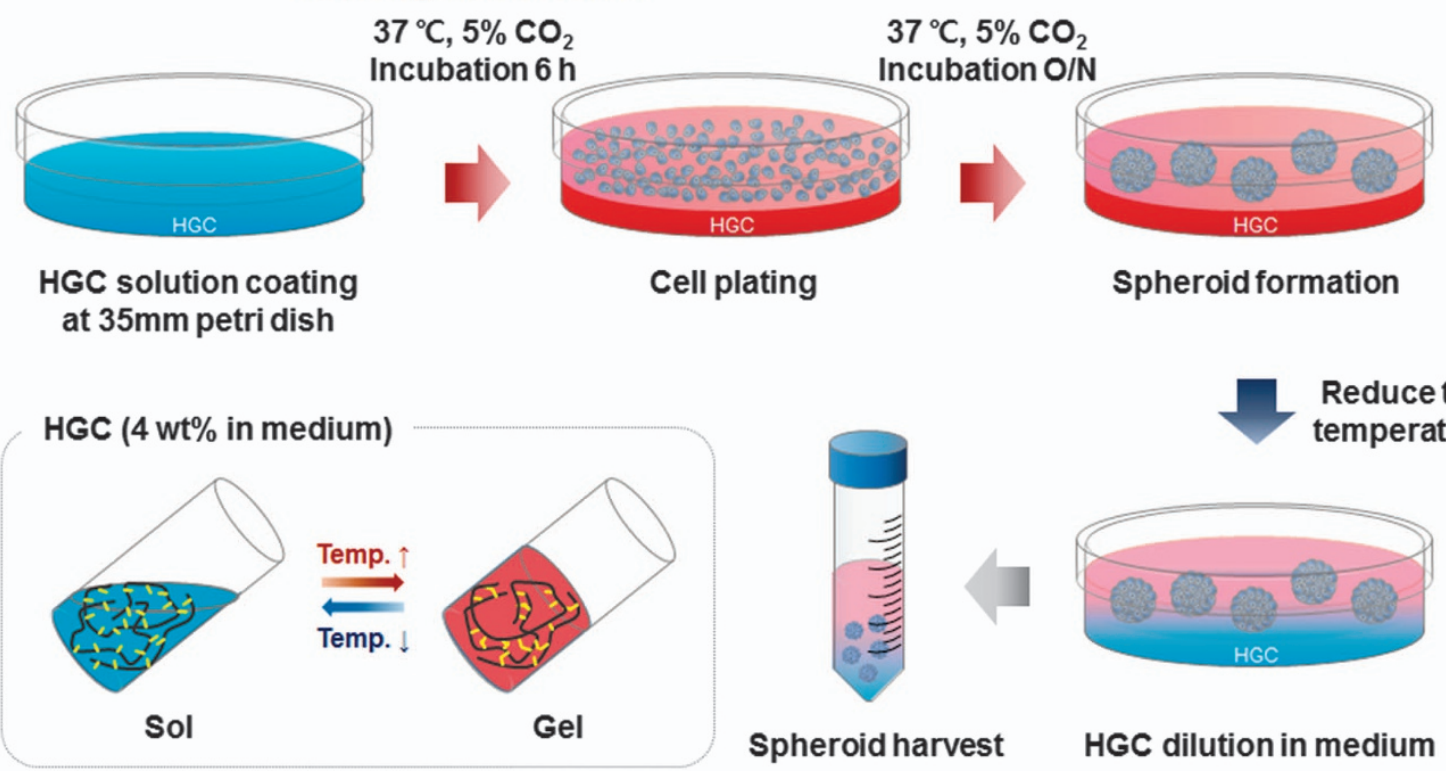

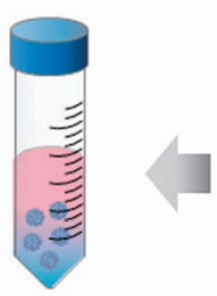

Spheroid harvest



HGC dilution in medium

Scheme 1 Schematic illustration of coating the surface of a cell culture plate using HGC and the process of spheroid formation on an HGC-coated plate.

of hydrogel-coated surfaces, increase stability in aqueous media for a longer period, and allow for the harvesting of 3D spheroids with high viability and intact surface proteins of spheroids by a simple temperature control method.

The objective of this study was to develop novel chitosan derivatives with enhanced non-adhesive and temperature-tunable physical properties that can allow for simple and effective 3D spheroid formation and culture (Scheme 1). $\mathrm{N}$-acylated glycol chitosans were developed as a new class of thermo-reversible hydrogels by using a simple $\mathrm{N}$-acylation reaction involving glycol chitosan (GC). Herein, we report the synthesis of a series of thermo-reversible $\mathrm{N}$-acyl glycol chitosans (NAGCs) and a facile method for the formation of 3D spheroids using various cells on a thermo-reversible hydrogel-coated culture plate.

\section{EXPERIMENTAL PROCEDURES}

\section{Materials}

GC $(D P \geqslant 200$; degree of acetylation $=9.3 \%)$ was purchased from Wako Pure Chemical Industries, Ltd. (Osaka, Japan). Acetic anhydride (99.5\%), propionic anhydride (99\%), butyric anhydride (98\%), valeric anhydride (97\%) and hexanoic anhydride (97\%) were obtained from Sigma-Aldrich (Saint Louis, MO, USA). Methanol and acetone were supplied by Samchun Pure Chemical Co. Ltd. (Seoul, Korea). Dialysis membrane tubing (Molecular weight cut-off: $10-12 \mathrm{kDa}$ ) was purchased from Spectrum Laboratories (Houston, TX, USA). All chemical reagents were of analytical grade and were used without further purification.

\section{Synthesis of NAGCs}

A series of NAGCs, including $\mathrm{N}$-acetyl glycol chitosan, $\mathrm{N}$-propionyl glycol chitosan, $\mathrm{N}$-butyryl glycol chitosan, $\mathrm{N}$-valeryl glycol chitosan and $\mathrm{N}$-hexanoyl glycol chitosan (HGC), were synthesized via the $\mathrm{N}$-acylation of GC with corresponding carboxylic anhydrides (acetic anhydride, propionic anhydride, butyric anhydride, valeric anhydride and hexanoic anhydride). In brief, GC $(1 \mathrm{~g})$ was dissolved in $125 \mathrm{ml}$ of distilled water, followed by dilution with $125 \mathrm{ml}$ of methanol. A predetermined amount of carboxylic anhydride was added drop-wise to the polymer solution. After continuous stirring at room temperature for $48 \mathrm{~h}$, the reaction product was precipitated with acetone and centrifuged to enable separation. Then, the product was dialyzed using a dialysis membrane (molecular weight cut-off: $10-12 \mathrm{kDa}$ ) against distilled water for 3 days to remove impurities, followed by lyophilization to obtain a solid white powder, ( $N$-acetyl glycol chitosan, $N$-propionyl glycol chitosan, $N$-butyryl glycol chitosan, $\mathrm{N}$-valeryl glycol chitosan and HGC).

\section{Characterization of NAGCs}

The chemical structures of NAGCs were characterized by ${ }^{1} \mathrm{H}-\mathrm{NMR}$ spectroscopy using an Avance III 600 spectrometer (Brucker, Germany) operating at $600 \mathrm{MHz}$. The polymer samples were dissolved in $\mathrm{D}_{2} \mathrm{O}$ at a concentration of $1 \mathrm{wt} \%$. The $\mathrm{D}_{2} \mathrm{O}$ peak at $\delta 4.65$ was used as a reference peak. The attenuated total reflectance Fourier transform infrared spectroscopy (ATR-FTIR) spectra of the NAGCs were recorded using a Nicolet iS5 spectrometer (Thermo Scientific, MA, USA). The analysis was performed with 16 scans gathered at a resolution of $4 \mathrm{~cm}^{-1}$ over a frequency range of $3800-750 \mathrm{~cm}^{-1}$ to confirm the chemical modification.

Thermo-reversible sol-gel transitions were determined by a tube tilting method with a temperature increment of $0.2^{\circ} \mathrm{C} \mathrm{min}{ }^{-1}$. Each sample was prepared by dissolving the polymer in phosphate-buffered saline at a given concentration $(\mathrm{pH} 7.4)$ at $4{ }^{\circ} \mathrm{C}$. The gelation temperature was determined by tilting the tube until no fluidity was observed after $30 \mathrm{~s}$. The gelation temperature was also measured by a rotating rheometer (TA Instruments, AR 1500ex, New Castel, DE, USA). The aqueous polymer solutions were placed between parallel plates with a diameter of $20 \mathrm{~mm}$ and a gap of $1000 \mu \mathrm{m}$. The frequency was optimized to $1 \mathrm{~Hz}$, which was determined in a frequency sweep experiment. A constant stress of $25 \mathrm{~Pa}$ was used for the measurements.

\section{Preparation of culture plates coated with GC and HGC}

GC or HGC (4 wt $\%$ ) was dissolved in Dulbecco's Modified Eagle's Medium (DMEM, Gibco, Big Cabin, OK, USA) and was maintained at $4{ }^{\circ} \mathrm{C}$. After the polymer was dissolved completely, $200 \mu \mathrm{l}$ of polymer solution was added to each $35 \mathrm{~mm}$ Petri dish (SPL Life Sciences Co., Ltd., Seoul, Korea) and spread evenly with a $1 \mathrm{ml}$ syringe. The GC- and HGC-coated dishes were incubated at $37^{\circ} \mathrm{C}$ and then used to cultivate spheroids.

\section{Quantitative stability test of GC and HGC}

GC and HGC were dissolved in $0.5 \mathrm{ml}$ of DMEM (4 wt $\%$ ) into vials and incubated for $30 \mathrm{~min}$ at $37^{\circ} \mathrm{C}$, and then $0.5 \mathrm{ml}$ DMEM was added into the GC- and HGC-containing vials. Then, the vials were again placed in the incubator. The incubation temperature was maintained at $37^{\circ} \mathrm{C}$ for 1 week. At predetermined time intervals $(0.5,1,2,4,8,14,24,36,48,72,120$ and $168 \mathrm{~h})$, DMEM was removed with a pipette and replaced with fresh DMEM. Every sample was weighed at each time interval. 


\section{Protein adsorption test}

Proteins were adsorbed onto GC-, HGC- and chitosan-coated surfaces with bovine serum albumin (BSA, Bovogen, Melbourne, Australia). GC- or HGC-coated surfaces were prepared by coating 24-well tissue culture plates with $100 \mu \mathrm{l}$ per well of a GC or HGC solution (4 wt\% in distilled water) as previously described. Chitosan (Sigma, St Louis, MO, USA) was dissolved in a $1 \%$ acetic acid solution to obtain a $1 \mathrm{wt} \%$ chitosan solution, and $100 \mu \mathrm{l}$ of a chitosan solution was added to each well of 24-well tissue culture plates, followed by air drying. Each well was neutralized with a $0.1 \mathrm{~N} \mathrm{NaOH}$ solution and washed with distilled water. After preparing polymer-coated surfaces, a BSA solution $\left(2 \mathrm{mg} \mathrm{ml}^{-1}\right)$ diluted in distilled water was added to each well of the polymer-coated plates. At the designated time, the solutions were obtained and the amounts of adsorbed protein were quantified using a bicinchoninic (BCA, Thermo Scientific, Rockford, IL, USA) assay.

\section{Isolation and culture of neonatal rat cardiomyocytes}

Neonatal rat cardiomyocytes were isolated from 1-2-day-old Sprague Dawley rats. In brief, neonatal rat hearts were excised and chopped in a $0.2 \%$ trypsin (Gibco) solution. The tissues were digested repeatedly in an enzyme cocktail that included $0.05 \%$ collagenase II (Worthington, Freehold, NJ, USA) $/ 0.06 \%$ pancreatin (Sigma). The cells were subsequently pre-plated in $100 \mathrm{~mm}$ tissue culture dishes to separate the attached non-cardiomyocytes. Then, unattached cardiomyocytes were collected and plated in collagen-coated tissue culture plates, after which the cells were incubated at $37^{\circ} \mathrm{C}$ in the presence of $5 \% \mathrm{CO}_{2}$. The culture medium consisted of DMEM supplemented with $10 \%$ newborn calf serum (Gibco) and $1 \%$ penicillin-streptomycin ( $\mathrm{P} / \mathrm{S}$, Gibco), and the medium was refreshed every $2-3$ days.

To compare the culture methods using GC or HGC, cardiomyocytes were plated at densities of $0.5 \times 10^{5}, 1 \times 10^{5}, 2 \times 10^{5}, 5 \times 10^{5}$ and $10 \times 10^{5}$ in each plate ( $35 \mathrm{~mm}$ un-coated Petri dishes and GC- and HGC-coated dishes). The morphologies of the cells were observed using an optical microscope (Olympus LX51, Olympus, Tokyo, Japan) at the indicated times. To determine the size distribution of the spheroids and the percentage of aggregated cells, each culture plate was photographed, and the images were arranged to obtain arrays of micrographs. After imaging, the number and width of the spheroids were calculated using cellSens Standard software (http://www.olympus-lifescience. com/en/software/cellsens), and the percentage of aggregated cells was analyzed using the ImageJ software program (http://imagej.nih.gov/ij/).

\section{Isolation and culture of chondrocytes}

Chondrocytes were isolated from the articular cartilage of New Zealand white rabbits. In brief, knee cartilage was cut with a sterile scalpel and transferred to a $100 \mathrm{~mm}$ Petri dish. The cartilage was washed with PBS containing P/S and chopped finely using a blade. Then, the tissue was incubated in DMEM containing $0.05 \%$ collagenase II at $37^{\circ} \mathrm{C}$ in the presence of $5 \% \mathrm{CO}_{2}$ until the matrix was digested. After overnight incubation, isolated chondrocytes were cultured in DMEM supplemented with 10\% fetal bovine serum (Gibco) and $1 \%$ penicillin. When the cells had been cultured from passage 0 to 1 , they were preserved via freezing and thawed before further experimentation.

Chondrocytes were cultured up to passage 3 , and the cells were plated into $35 \mathrm{~mm}$ Petri dishes and HGC-coated dishes at densities of $0.5 \times 10^{5}, 1 \times 10^{5}$, $2 \times 10^{5}, 5 \times 10^{5}$ and $10 \times 10^{5}$. The morphology of the cells was confirmed via microscopy on day 2, and the distribution of the number and size of cell spheroids was calculated using the cellSens Standard software.

To measure the effect of HGC's degree of acylation (DA) on spheroid formation, we prepared HGC with $\mathrm{DA}=32 \%$ (DA 32), $\mathrm{DA}=36.5 \%$ (DA 36.5) and $\mathrm{DA}=40 \%$ (DA 40). Each HGC solution was dissolved in DMEM medium at an adequate concentration for a sol-gel transition near body temperature (concentration; DA $32=5 \mathrm{wt} \%$, DA $36.5=4 \mathrm{wt} \%$, DA $40=2 \mathrm{wt} \%$ ). The cells were seeded on HGC-coated dishes at $5 \times 10^{5}$ cells per plate. After $24 \mathrm{~h}$ of incubation, the spheroids were analyzed and calculated.

\section{Cell proliferation and viability test}

The cytotoxicity of GC and HGC was estimated using the 3-(4,5-dimethylthiazol-2-yl)-2,5-diphenyl tetrazolium bromide (MTT, Sigma) assay. In brief, cardiomyocytes were plated in collagen-coated 96-well tissue culture plates
(Corning, Saint Louis, MO, USA) at a density of $2 \times 10^{4}$ per well in DMEM containing $10 \%$ newborn calf serum and $1 \% \mathrm{P} / \mathrm{S}$. After $24 \mathrm{~h}$ of incubation, the culture medium was replaced with DMEM or DMEM containing $1 \mathrm{wt} \%$ GC or $1 \mathrm{wt} \%$ HGC. On days 1, 3, 5 and 7 of culture in medium containing GC or HGC, $20 \mu \mathrm{l}$ of MTT solution ( $5 \mathrm{mg} \mathrm{ml}^{-1}$ in PBS) was directly added to each culture well, and the plates were incubated at $37^{\circ} \mathrm{C}$ for $2 \mathrm{~h}$. After removing the culture medium, $150 \mu \mathrm{l}$ of dimethyl sulfoxide (DMSO, Sigma) was added to each well, and the plates were shaken until the formazan crystals dissolved. The absorbance was measured at $540 \mathrm{~nm}$ using a microplate reader (SpectraMax M3, Molecular devices, Sunnyvale, CA, USA).

Cell viability was determined with a live/dead assay kit (Abcam, Cambridge, UK). To perform viability assays in cardiomyocytes that were cultured with DMEM containing $1 \mathrm{wt} \%$ GC or $1 \mathrm{wt} \%$ HGC, $12 \mathrm{~mm}$ collagen-coated cover slips were placed in the wells of a 24-well tissue culture plate, and cells were seeded in these plates at a density of $1 \times 10^{5}$ per well in DMEM. After 1 day of incubation at $37^{\circ} \mathrm{C}$, the medium was exchanged with DMEM containing $1 \mathrm{wt}$ $\%$ GC or $1 \mathrm{wt} \%$ HGC. On day 3 , the media were replaced with a staining solution, and the samples were then incubated for $15 \mathrm{~min}$ at $37^{\circ} \mathrm{C}$ and examined via fluorescence microscopy. The viability of aggregates on GC- or HGC-coated dishes was also measured by live/dead assay and observed using laser-scanning confocal microscopy (LSM 710, Carl Zeiss, Thornwood, NY, USA).

\section{SEM analysis}

Surface analysis was performed via scanning electron microscopy (SEM; JEOL JSM-7000F, Peabody, MA, USA). GC- or HGC-coated Petri dishes were divided into $5 \times 5 \mathrm{~mm}$ sections, and an un-coated Petri dish was used as the control. These samples were placed in 6-well tissue culture plates containing $4 \mathrm{ml}$ of cardiomyocyte culture media. After 1 and 3 days, samples were collected and freeze-dried for $24 \mathrm{~h}$. The samples were subsequently treated with a thin layer of platinum after they were attached to carbon tape.

The morphology of the aggregated cells on HGC-coated dishes was observed under an ultra-high resolution SEM (S-4800, HITACHI, Tsukuba, Japan). Cardiomyocytes were plated in HGC-coated dishes at a density of $5 \times 10^{5}$ cells per plate. After $24 \mathrm{~h}$, the spheroids were fixed in a $2.5 \%$ solution of glutaraldehyde (EMS, Fort Washington, PA, USA) in PBS at $4{ }^{\circ} \mathrm{C}$ and washed twice using PBS. The samples were post-fixed with $1 \%$ osmium tetroxide (EMS) on ice for $1 \mathrm{~h}$ and dehydrated stepwise using a graded ethanol series up to $100 \%$. The samples were then treated three times with hexamethyldisilazane and dried at room temperature. Before observation, the samples were coated with osmium.

\section{Morphology and beating behavior of cardiomyocytes}

To confirm the effect of medium containing GC and HGC on the beating ability of cardiomyocytes, cells were plated in 24-well tissue culture plates containing collagen-coated coverslips at a cell density of $1 \times 10^{5}$ per well. After $24 \mathrm{~h}$ of incubation, the medium was exchanged with DMEM containing $1 \mathrm{wt} \%$ GC or $1 \mathrm{wt} \%$ HGC. On day 3 , the beating ability of the cardiomyocytes was recorded via optical microscopy.

To observe the beating ability of long-term-cultured cardiomyocyte spheroids in GC- and HGC-coated dishes, cardiomyocytes were seeded in GC- or HGC-coated dishes at a concentration of $5 \times 10^{5}$ per well. The plates were incubated at $37^{\circ} \mathrm{C}$ in the presence of $5 \% \mathrm{CO}_{2}$. After 10 days of incubation, the behavior of the spheroids in hydrogel-coated dishes was recorded via optical microscopy.

\section{Time-lapse imaging of cardiomyocyte spheroid formation}

The cardiomyocytes were seeded in HGC-coated dishes at a cell density of $5 \times 10^{5}$ per plate with DMEM supplemented with $10 \%$ newborn calf serum and $1 \% \mathrm{P} / \mathrm{S}$. Then, the plate was incubated at $37^{\circ} \mathrm{C}$ in the presence of $5 \% \mathrm{CO}_{2}$. Images were obtained using NIS-element BR software (Nikon, Japan) and were collected every $3 \mathrm{~min}$ for $24 \mathrm{~h}$.

\section{Immunofluorescence staining}

Cardiomyocyte spheroids that formed on un-coated Petri dishes or GC/HGC hydrogel-coated dishes were collected on day 7 through pipetting and fixed in 
a

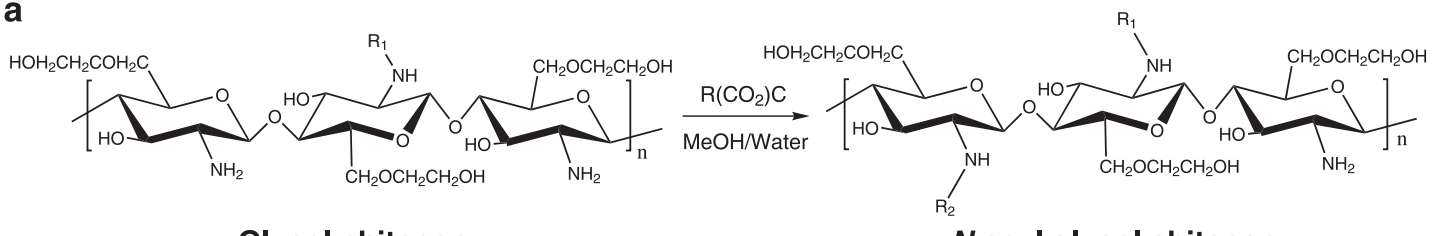

Glycol chitosan

N-acyl glycol chitosan

$\left(\mathrm{R}_{1}=\mathrm{H}\right.$ or $\mathrm{COCH}_{3}, \mathrm{R}_{2}=$ acyl group $)$

b



C

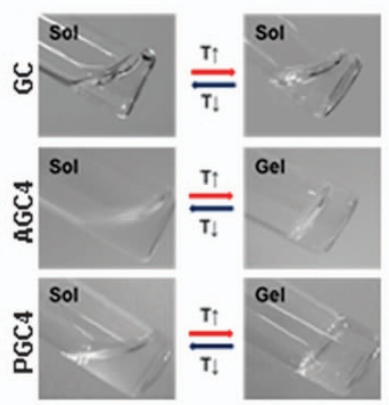

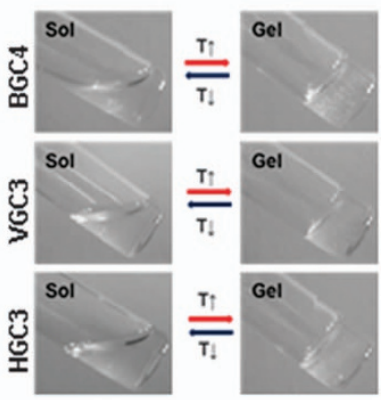

d

GC

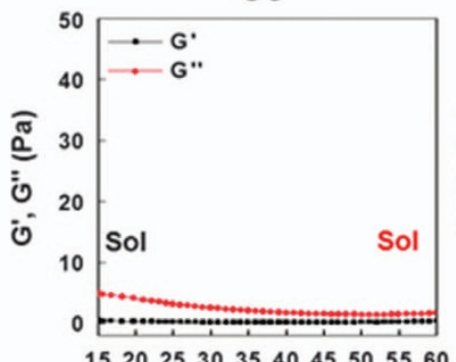

Temperature $\left({ }^{\circ} \mathrm{C}\right)$

\section{BGC4}

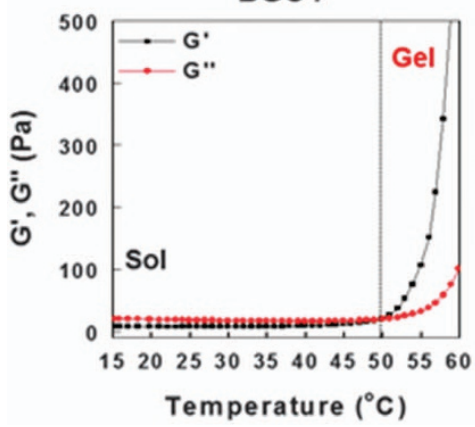

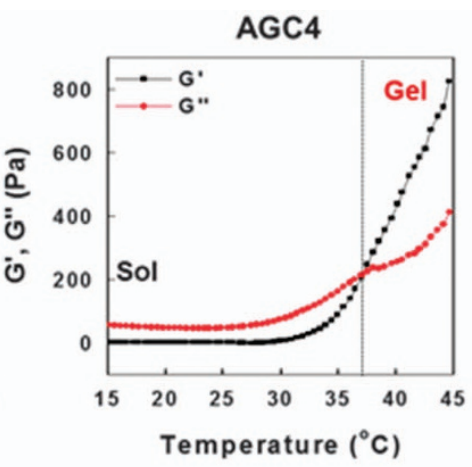

VGC3

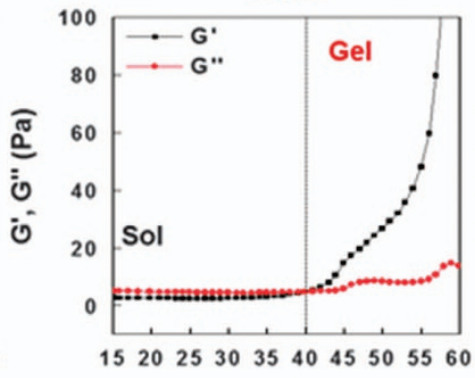

Temperature $\left({ }^{\circ} \mathrm{C}\right)$

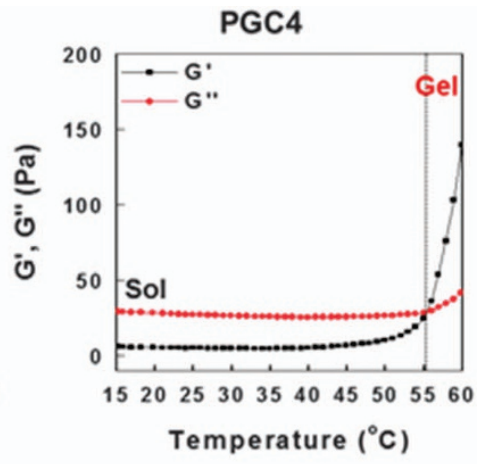

HGC3

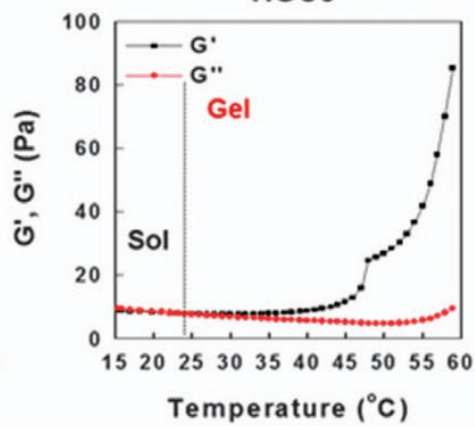

Figure 1 Synthesis and characterization of thermo-reversible sol-gel transition temperatures of NAGCs. (a) Chemical structure of $N$-acylation reaction of GC. (b) Thermo-reversible sol-gel transition temperatures of NAGCs and (c) images of thermo-reversible sol-gel transitions of NAGCs. (d) Temperature-dependent changes in the elastic $\left(\mathrm{G}^{\prime}\right)$ and viscous $\left(\mathrm{G}^{\prime \prime}\right)$ moduli of aqueous solutions (4 wt\%) of GC and NAGCs.

$4 \%$ paraformaldehyde at $4{ }^{\circ} \mathrm{C}$. The samples were subsequently embedded in Tissue-Tekcryomold (Sakura Finetek, Tokyo, Japan) with FSC 22 Clear (Leica, Tokyo, Japan), and $10 \mu \mathrm{m}$ sections were prepared. For immunofluorescence staining, the specimens were permeabilized with PBS containing $0.5 \%$ Triton X-100 (Sigma) for $5 \mathrm{~min}$ at room temperature and washed three times with $0.1 \%$ Triton X-100 in PBS. After washing, the samples were blocked with PBS containing 2\% BSA (Bovogen, Victoria, Australia) and 0.1\% Triton-X 100 and incubated for $20 \mathrm{~min}$. The blocking solution was removed via suction, and the samples were incubated overnight with primary antibodies at $4{ }^{\circ} \mathrm{C}$.
The primary antibodies used in these experiments were rabbit anti-cardiac Troponin I (cTnI, 1:100 dilution; Abcam) and mouse anti-Connexin 43 (Cx43, 1:100 dilution; Abcam). After incubation, the samples were washed with $0.1 \%$ Triton X-100 in PBS three times and then incubated for $45 \mathrm{~min}$ with donkey anti-rabbit IgG H\&L DyLight 488 (1:300 dilution; Abcam) and donkey anti-mouse IgG H\&L Alexa Fluor594 (1:300 dilution; Abcam). The slides were finally mounted with Fluoroshield mounting medium with 4',6-diamidino-2-phenylindole (Abcam) and analyzed via laser-scanning confocal microscopy. 


\section{Formation of spheroids for ADSCs/cardiomyocytes co-culture}

Neonatal rat cardiomyocytes were co-cultured with adipose-derived stromal cells (ADSCs). ADSCs were isolated from canine abdominal fat tissue. In brief, samples of fat were cut into slices and then chopped. The samples were then treated with $0.01 \%$ collagenase type I (Wako) for $8 \mathrm{~h}$ at $37^{\circ} \mathrm{C}$. After digestion, the cells were collected via centrifugation at 1500 r.p.m. for $15 \mathrm{~min}$. The cell pellet was then washed with PBS and centrifuged again at 1500 r.p.m. for $5 \mathrm{~min}$. The obtained cells were filtered through a $0.22 \mu \mathrm{m}$ strainer to remove debris. ADSCs were cultivated in DMEM supplemented with $10 \%$ fetal bovine serum and $1 \% \mathrm{P} / \mathrm{S}$, and passage 5 was selected. Before using the cells for co-cultivation, the ADSCs were stained with a Vybrant CM-DiO cell-labeling solution (Invitrogen, Carlsbad, CA, USA), and the cardiomyocytes were stained with a Vybrant CM-DiD cell-labeling solution (Invitrogen). Then, the ADSCs were seeded into HGC-coated dishes at a density of $5 \times 10^{5}$ cells per well. After $24 \mathrm{~h}$ of incubation, the cardiomyocytes were plated into these coated dishes at a density of $2 \times 10^{5}, 5 \times 10^{5}, 10 \times 10^{5}$ and $15 \times 10^{5}$ cells per well.

One day after the cardiomyocytes were plated, spheroids were collected via pipetting. Then, the cells were fixed in $4 \%$ paraformaldehyde at $4{ }^{\circ} \mathrm{C}$ and embedded in Tissue-Tekcryomold with FSC 22 Clear and cut into sections measuring $10 \mu \mathrm{m}$ thick. The slides were washed with deionized water and treated with Fluoroshield mounting medium containing 4',6-diamidino-2phenylindole. The samples were examined using a confocal laser-scanning microscope.

\section{Statistical analysis}

Quantitative data are expressed as means \pm s.d. Statistical analyses were performed with one-way analysis of variance using SPSS software (SPSS Inc., Chicago, IL, USA). A value of $P<0.05$ was considered statistically significant.

\section{RESULTS AND DISCUSSION}

\section{Synthesis of NAGCs}

We selected $N$-acylation with GC as a chemical modification because $\mathrm{N}$-acylation can easily present amphiphilic characteristics through hydrophobic interactions. ${ }^{13}$ NAGCs were synthesized with various carboxylic anhydrides (acetic, propionic, valeric and hexanoic anhydrides) in a simple one-step reaction procedure under mild conditions at room temperature (Figure 1a). Their synthetic results are summarized in the Supplementary Information (Supplementary Table S1 and Supplementary Figure S1). As expected, GC was not thermo-responsive and existed in a flowing sol state, even at an elevated temperature. However, aqueous solutions of the NAGCs exhibited a phase transition from a flowing sol state to a non-flowing gel state as the temperature increased.

The chemical composition and structures of the NAGCs were characterized through ${ }^{1} \mathrm{H}-\mathrm{NMR}$ and fourier transform infrared spectroscopy measurements. The ${ }^{1} \mathrm{H}-\mathrm{NMR}$ spectra of representative NAGCs are shown in Supplementary Figure Sla. The peak at 3.4-3.8 p.p.m. arose from $\mathrm{H} 2$ to $\mathrm{H} 8$ of the glucopyranosyl ring, and the peak at $2.74 \mathrm{ppm}$ revealed protons of the primary amine residue $\left(-\mathrm{NH}_{2}\right)$. The methyl protons of the acetyl group were observed at 2.02 p.p.m. After $N$-acylation, several new proton peaks from each acyl group appeared. For the $\mathrm{N}$-propionyl glycol chitosans, the new proton peaks at 0.7 and 2.2 p.p.m. were assigned to $-\mathrm{CH}_{3}$ and $-\mathrm{CO}-\mathrm{CH}_{2}$ of the propionyl groups, respectively. For the $N$-butyryl glycol chitosans and $\mathrm{N}$-valeryl glycol chitosans, the new peaks at 1.5 and 1.2 p.p.m. were assigned to $-\mathrm{CO}-\mathrm{CH}_{2}-\mathrm{CH}_{2}-$ and $-\mathrm{CO}-\mathrm{CH}_{2}-\mathrm{CH}_{2}-\mathrm{CH}_{2}$, respectively. The HGCs exhibited new peaks at 2.31, 1.62, 1.32 and 0.89 p.p.m., which resulted from $\mathrm{N}$-hexanoyl residues (-CO- $\mathrm{CH}_{2}$, $-\mathrm{CO}-\mathrm{CH}_{2}-\mathrm{CH}_{2}-\mathrm{CH}_{2}-\mathrm{CH}_{2}-\mathrm{CH}_{3}$, - $\mathrm{CO}-\mathrm{CH}_{2}-\mathrm{CH}_{2}-\mathrm{CH}_{2}-\mathrm{CH}_{2}-\mathrm{CH}_{3}$ and $-\mathrm{CO}-\mathrm{CH}_{2}-\mathrm{CH}_{2}-\mathrm{CH}_{2}-\mathrm{CH}_{2}-\mathrm{CH}_{3}$, respectively). Based on these assignments, the average degree of acylation (DA) of the NAGCs was calculated by comparing the integrated signal area of the protons of the glucopyranosyl ring with that of the acyl groups. The degree of acetylation for GC itself was observed to be $9.3 \%$. The DA values for the NAGCs were in the range of $73.1-91.6 \%$ for $N$-acetyl glycol chitosans, $48.3-74.5 \%$ for $N$-propionyl glycol chitosans, $36.3-61.4 \%$ for $N$-butyryl glycol chitosans, $26.7-48.0 \%$ for $N$-valeryl glycol chitosans and $19-36.5 \%$ for HGCs. The DA was easily controlled by changing the feed molar ratio of carboxylic anhydride, which exhibited a proportionally increasing trend. The NAGCs with DA values above or below the DA range were not water soluble or did not show any thermo-reversible properties across the range of experimental concentrations and temperatures used in this study. The fourier transform infrared spectroscopy spectra confirmed the chemical structure of the GC derivatives (Supplementary Figure S1b). The peak at $1655 \mathrm{~cm}^{-1}$ represents the carbonyl stretching vibration, and the absorption peak at $2850-2930 \mathrm{~cm}^{-1}$ was ascribed to the $\mathrm{C}-\mathrm{H}$ stretching of methylene and methyl groups. For the NAGCs, the peak at $1570 \mathrm{~cm}^{-1}$, which corresponds to the bending of GC amino groups, nearly disappeared, whereas new absorption peaks resulting from the bending vibration of NAGC amide II bonds were observed at $1555 \mathrm{~cm}^{-1}$.

\section{Properties of thermo-reversible NAGCs}

All of the NAGCs exhibited thermo-reversible sol-gel transition behavior that was concentration dependent (Figure $1 \mathrm{~b}$ and $\mathrm{c}$ ). The range of concentrations over which the sol-gel transition occurred could be varied based on the type and DA. In the case of a $3 \mathrm{wt} \%$ HGC solution in PBS, the sol-gel transition temperature $\left(T_{\text {gel }}\right)$ was observed to be $42.0 \pm 1^{\circ} \mathrm{C}$. The $T_{\text {gel }}$ points for 4 and $5 \mathrm{wt} \% \mathrm{HGC}$ solutions were $32.3 \pm 0.6^{\circ} \mathrm{C}$ and $27.3 \pm 0.6{ }^{\circ} \mathrm{C}$, respectively. No sol-gel transition was observed below the designated range of concentrations. Alternatively, a higher concentration range led to premature gelation at room temperature. The $T_{\text {gel }}$ point for all of the NAGCs decreased as the concentration increased. Notably, those NAGCs produce transparent hydrogels and maintain their transparency, even above $70^{\circ} \mathrm{C}$. In contrast, other conventional thermo-reversible hydrogels, including poly( $N$-isopropylacrylamide) derivatives, poly(ethylene oxide)-based block copolymers and polyphosphazenes, are known to change from a transparent gel to an opaque gel as the temperature is continuously increased. ${ }^{14}$ Importantly, the NAGCs can also undergo a sol-gel transition over a relatively lower concentration range. All of the NAGCs form gels over a concentration range of 3-7 wt $\%$, but the typical block copolymer-based systems undergo a sol-gel transition only above $15 \mathrm{wt} \%$ and show limited stability under dilute conditions. ${ }^{14}$ These results suggest an optimal coating condition on Petri dishes for the formation of cell aggregates on the dish surface.

The thermo-reversible gelation of the NAGCs was also studied by dynamic rheological methods (Figure 1d). The viscoelastic parameters $\left(G^{\prime}\right.$ and $\left.G^{\prime \prime}\right)$ of the NAGC hydrogels were investigated as a function of temperature $\left(15-60^{\circ} \mathrm{C}\right)$. For $\mathrm{GC}, \mathrm{G}^{\prime}$ was lower than $\mathrm{G}^{\prime \prime}$ at all temperatures tested. In contrast, for the NAGC hydrogels, $G^{\prime \prime}$ dominated $\mathrm{G}^{\prime}$ at lower temperatures, exhibiting the behavior of a viscous fluid (sol). When the temperature increased above a certain temperature, the elastic modulus $\left(G^{\prime}\right)$ increased rapidly. The higher rate of increase of $G^{\prime}$ led to a crossover with $G^{\prime \prime}$ at a certain temperature (defined as the gel point), indicating the transition from a viscous behavior-dominated liquid phase to an elastic behaviordominated solid phase. This negatively thermo-responsive gelation may result from the formation of physical crosslinks between the hydrophobic acyl groups. The thermo-reversible sol-gel transition properties could be well modulated between 22 and $73^{\circ} \mathrm{C}$ by changing various factors, such as the DA, the type of acyl groups and the polymer concentration. Among the various NAGCs, HGC3 showed the greatest thermo-sensitivity and displayed a relatively lower $T_{\text {gel }}$, 
a

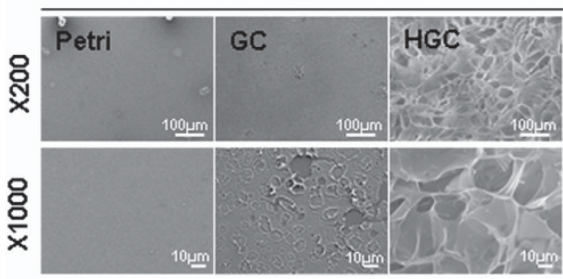

b

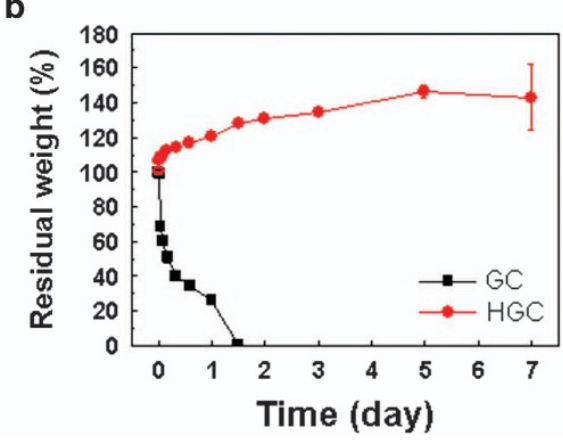

Day 3

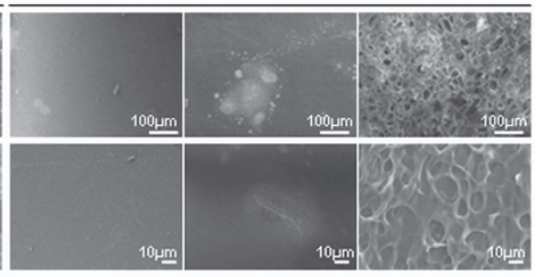

C

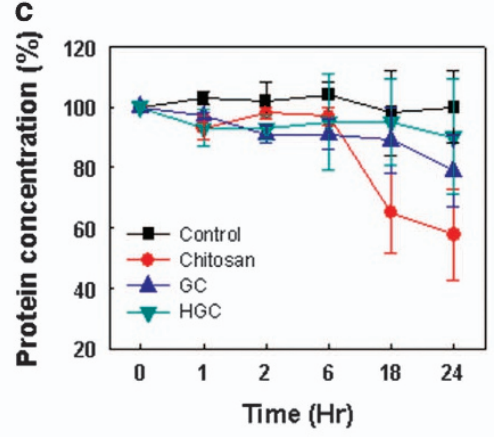

Figure 2 (a) SEM images of cell culture plate coated with GC and HGC after soaking in DMEM culture medium for 1 and 3 days. (b) Stability test of GC and $\mathrm{HGC}$ in aqueous media at $37^{\circ} \mathrm{C}$. (c) Adsorption of BSA on chitosan-, GC- and HGC-coated plates in PBS containing BSA for $24 \mathrm{~h}$.

even over a lower concentration range (3-5 wt \%) and with a lower DA (36.5\%) compared with other NAGCs. An aqueous solution of $4 \mathrm{wt} \%$ HGC3 exhibited a $T_{\text {gel }}$ of $\sim 32^{\circ} \mathrm{C}$, which is below body temperature, and it could therefore be conveniently used to introduce a hydrogel coating onto culture dishes based on the sol-gel transition property. The strong thermo-sensitivity of HGC3 could also render the hydrogel layer more stable and prevent premature dissolution for longer culture duration.

\section{Cytotoxicity of $\mathrm{N}$-hexanoyl glycol chitosan}

The potential cytotoxicity of HGC was evaluated by measuring mitochondrial metabolic activity and through fluorescence staining in a live/dead assay of cardiomyocytes cultured in the presence of HGC (Supplementary Figure S2). Mitochondrial activities were measured by the MTT assay and were calculated by normalizing the absorbance of the samples at $540 \mathrm{~nm}$ to that of a control without HGC in the medium (Supplementary Figures S2a). The mitochondrial activity of cardiomyocytes cultured in the presence of HGC was higher than that of the control over 7 days in culture. There were no significant differences between the mitochondrial activities of cardiomyocytes cultured with GC or HGC. As shown in Supplementary Figure S2b, high cell viability was observed for all groups on day 3 . A video recording showing the beating behavior of cardiomyocytes is presented in Supplementary Movie S1. The beating profile of cardiomyocytes cultured with basal medium and medium containing HGC was regular. However, the beating of cardiomyocytes cultured with medium containing GC was largely reduced. Although the actual mechanism underlying the effect of HGC against cytotoxicity is unknown, the present findings indicate that as prepared, HGC is fairly cytocompatible and non-toxic in nature and provides a favorable environment for cell culture.

Surface morphologies, stability and protein adsorption of polymer coated plates

To coat HGC on the surface of a cell culture plate, adequate amounts of HGC solution (4 wt $\%$ ) in a sol state were spread on the plate at room temperature, followed by incubation at $37^{\circ} \mathrm{C}$ to permit thermogelation. The GC was used as a control group. The surface morphologies of the coated dishes were analyzed via SEM (Figure 2a). On day 1, the SEM images showed that the dishes were surface-coated with GC or HGC. On day 3, a significant amount of GC was washed out and removed from the surface. In contrast, the HGC coating layer remained intact, as observed on day 1 . After 3 days in the culture medium, most of the GC coating layer was lost due to dissolution. However, the HGC coating was well preserved. We also quantitatively evaluated the stability of GC and HGC in aqueous media (Figure 2b). For GC, only $26 \%$ of the original weight remained after 1 day of incubation in the medium. After $36 \mathrm{~h}$ of incubation, no remaining GC was observed. In contrast, HGC showed good stability in aqueous media even after 7 days incubation due to its enhanced physical stability, a result of effective thermogelation.

With respect to the adsorption of purified BSA, the HGC-coated plate showed the lowest adsorption amount and the GC-coated plate showed moderate adsorption. The chitosan-coated plate showed the highest adsorption amount, which increased over time (Figure 2c). Positive charges on the surface of the chitosan-coated plate could help adsorb BSA. However, in our experiment, the adsorption amount of BSA on the HGC-coated plate was far less than that on the chitosancoated plate. Binding with cells on the substrate can be controlled by adhesion molecules. ${ }^{15}$ Chitosan can bind the cell adhesion molecules in the medium and present them to the cells for adhesion. This phenomenon may increase the probability of cell binding to the surface of chitosan over time in culture. The cell adhesion molecule binding capacity of chitosan can be controlled by the structural parameters of chitosan, particularly the number of free amino groups. ${ }^{16}$ HGC modified with acylation possesses fewer amino groups along the chain. The cell adhesion molecules and cell binding affinity of HGC would be lower than that of chitosan. Thus, HGC can provide a more suitable environment for the formation of cell spheroids and long-term culture of cell spheroids than that provided by chitosan. 

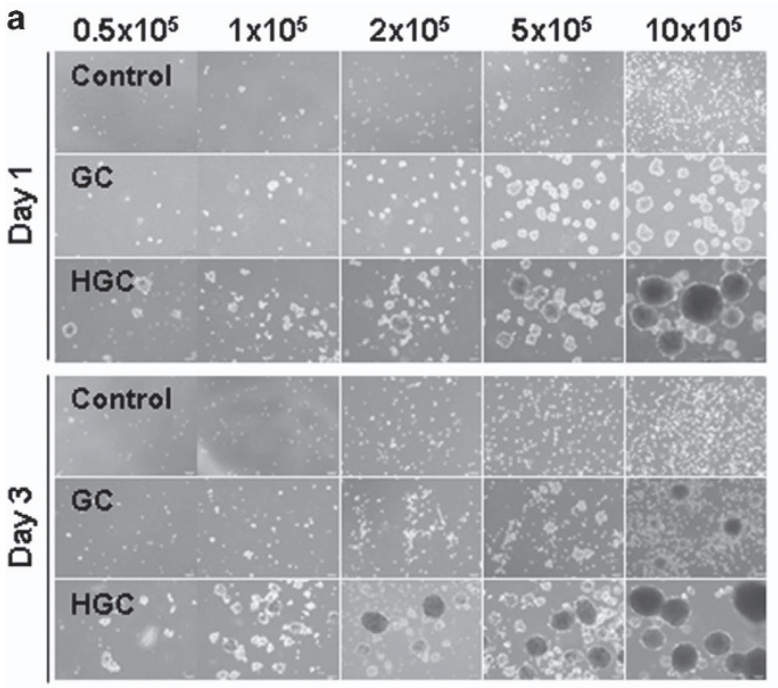

Scale bar; $100 \mu \mathrm{m}$ b
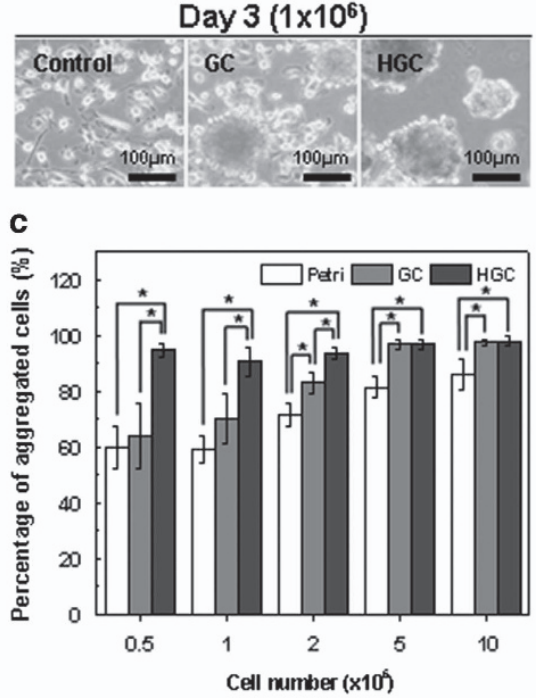

d
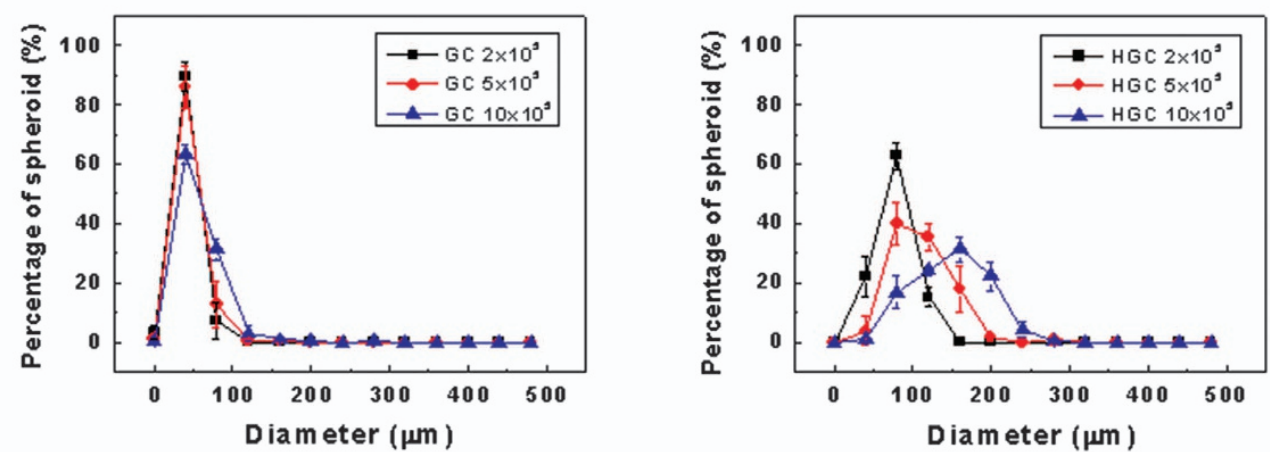

e

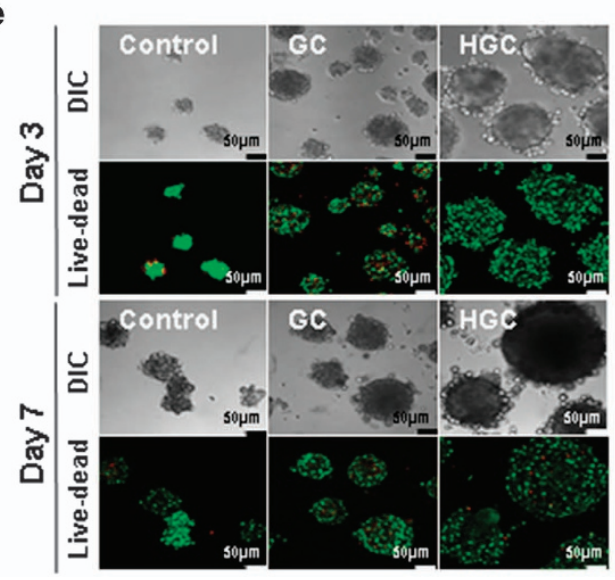

f

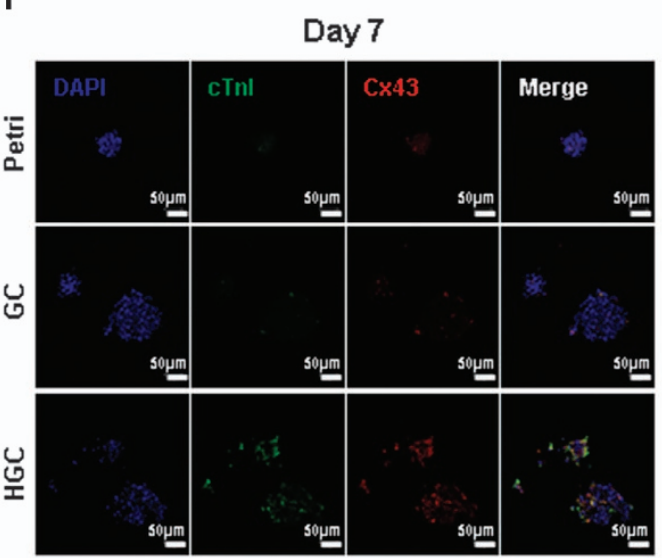

Figure 3 Cardiomyocytes cultured on un-coated plate and GC- or HGC-coated plate. (a) The morphologies of cardiomyocytes at 1 and 3 days. (b) Magnified images of cardiomyocytes on day 3. (c) Percentage of aggregated cardiomyocytes cultured on GC- and HGC-coated plates on day 1. (d) Distribution of the diameter and number of spheroids that formed on GC- and HGC-coated plates. (e) Live/dead assay of cardiomyocyte spheroids on days 3 and 7. (f) Immunofluorescence staining of $\mathrm{cTnl}$ and $\mathrm{Cx} 43$ in cardiomyocyte spheroids cultured on uncoated plates and on GC- and HGC-coated plates after cultivation for 7 days.

\section{Formation and harvesting of spheroids}

In the initial experiment, cardiomyocytes were cultured on GC- and HGC-coated surfaces for 3 days to study cell behavior at different seeding densities (Figure 3). On day 1, the cardiomyocytes on un-coated plates were suspended individually in the medium, without any significant aggregation, even at the highest seeding density of $10 \times 10^{5}$ per well. In contrast, on the GC- or HGC-coated plates, significant cell aggregation was observed on day 1. In particular, HGC led to aggregation even at the lowest cell concentration $\left(0.5 \times 10^{5}\right.$ per well). On day 3 , the cells on un-coated plates were attached separately to the 
bottom of the dishes at all densities. The spheroids present on GC-coated plates adhered to the bottom and showed partial disassembly and spreading behavior. In contrast, the spheroids that formed on HGC-coated dishes maintained their size and shape and did not show any attachment to the surface over more than 3 days (Figure $3 \mathrm{a}$ and $\mathrm{b}$ ). The process of cardiomyocyte spheroid formation on HGC-coated dishes was captured by a video camera with a built-in microscope and is shown in Supplementary Movie S2. In addition, the spheroid harvesting process on HGC-coated dishes was captured by a video camera and is shown in Supplementary Movie S3. The cells were plated in an HGC-coated dish and incubated at $37^{\circ} \mathrm{C}$. Spheroids were formed within $24 \mathrm{~h}$ and were easily harvested by pipetting. Moreover, spheroids on the HGC-coated plates could be harvested by temperature reduction by exploiting the thermo-sensitivity of HGC. To determine whether spheroid harvesting by temperature reduction is invasive to spheroids, we compared the morphology observed before harvesting spheroids to that observed after. There was no significant morphological change at $4{ }^{\circ} \mathrm{C}$ after 5 or $10 \mathrm{~min}$ (Supplementary Figure S3). In addition, when spheroids cultured in the HGC-coated dish for 3 days were transferred to a normal cell culture dish, the spheroids attached and spread on the bottom of the dish within $24 \mathrm{~h}$ (Supplementary Figure S4). These results indicate that spheroid harvesting by temperature reduction may not affect cell adhesion and growth.

We also observed the morphology of the aggregated cardiomyocytes that formed on the HGC-coated dishes by SEM (Supplementary Figure S5). The SEM images demonstrated that the cell aggregates possessed a spherical and compact structure. In this study, the GC surface became increasingly cell adherent, in contrast to the HGC surface, because of the weak stability of the coating layer. However, a coating layer of HGC with a strong thermogelling property remained intact for more than 3 days, thereby preventing cell-substrate interactions but enhancing cell-cell interactions. Thus, HGC can provide a more suitable environment for the formation of cell aggregates and their long-term culture without attachment to the surface.

In addition, we compared the spheroid-forming efficacy of three types of HGC-coated plates, each having different DA (DA $32=\mathrm{HGC}$ with DA of 32\%; DA $36.5=$ HGC with DA of $36.5 \%$; DA $40=$ HGC with DA of $40 \%$ ) for the optimal condition of HGC (Supplementary Figure S6). After 1 day of culture, spheroids were formed on all groups (Supplementary Figure S6a). The proportions of aggregated cells on the surface of DA 32, DA 36.5 and DA 40 were $98.4 \pm 0.8 \%$, $98.4 \pm 0.8 \%$ and $95.3 \pm 0.7 \%$, respectively (Supplementary Figure S6b), and the numbers of spheroids formed on the plates were statistically similar (DA $32=2483 \pm 1458.9$ cells per dish, DA $36.5=2445 \pm 149.9$ cells per dish, DA $40=2638 \pm 717.3$ cells per dish) (Supplementary Figure S6c). There were no statistically significant differences between the average sizes of the spheroids formed on the HGC-coated dishes (DA $32=67.8 \pm 23.9 \mu \mathrm{m}, \mathrm{DA} 36.5=94.1 \pm 31.7 \mu \mathrm{m}$ and DA $40=80.6 \pm 31.2 \mu \mathrm{m}) \quad$ (Supplementary Figure S6d). These results indicate that DA does not have a significant effect on spheroid formation. However, when the aqueous solutions of HGC with DA 32 (5 wt \%) and DA 40 (2 wt\%) were used for spheroid culture, the spheroids showed lower stability than those formed in the aqueous solution of HGC with DA 36.5 (data not shown); therefore, the HGC solution with DA $36(4 \mathrm{wt} \%)$ is the most suitable for spheroid culture.

A higher proportion of cells formed spheroids on the surface of HGC than on uncoated Petri dishes and GC-coated dishes after 1 day (Figure $3 \mathrm{c}$ ). The proportion of cells that became incorporated into spheroids (efficiency of spheroid formation) on the surface of the uncoated Petri dishes was only $60 \pm 7.5 \%$ at the lowest cell density $\left(0.5 \times 10^{5}\right.$ cells per well $)$ and increased to $86 \pm 5.5 \%$ at the highest cell density $\left(10 \times 10^{5}\right.$ cells per well), whereas the proportion of cells incorporated into spheroids on the surface of HGC-coated dishes was $95 \pm 2.5 \%$ at the lowest cell density. Nearly all of the cells on the surface of HGC-coated plates formed spheroids at all cell densities. The proportion of cells that formed spheroids on the surface of GC-coated dishes was higher than that on the uncoated Petri dishes but lower than that on the HGC-coated plates. After 3 days of cell plating, the sizes of the spheroids in GC- or HGC-coated dishes were analyzed at various cell densities $\left(2 \times 10^{5}, 5 \times 10^{5}\right.$ and $10 \times 10^{5}$ cells per well; Figure $3 \mathrm{~d}$ ). The diameters of the spheroids on the GC surface were determined using an inverse phase contrast microscope and ranged from 40 to $120 \mu \mathrm{m}$. Increasing or decreasing the cell density did not affect the spheroid size. In contrast, the diameters of the spheroids formed on the HGC surface ranged from 40 to $250 \mu \mathrm{m}$, and increasing the cell density appeared to increase the average spheroid size. As a result, the average spheroid size could be controlled by varying the cell density. In addition to cardiomyocytes, we investigated the formation of spheroids using chondrocytes (Supplementary Figure S7). On day 2, chondrocytes successfully aggregated on the HGC surface, and the diameter of the spheroids ranged from 40 to $280 \mu \mathrm{m}$. These observations are consistent with those obtained with cardiomyocytes. In addition, HGC-coated dishes provided a suitable environment for long-term culture (30 days) without attachment of chondrocyte aggregates on the surfaces.

To understand the fate of the cells in each spheroid, we performed live and dead cell assays through staining with a live/dead assay kit on days 3 and 7 (Figure 3e). A greater number of dead cells is typically expected in the central region of spheroids because nutrient and oxygen uptake by cells in the inner core of spheroids may be reduced because of the limitation of diffusion. ${ }^{17}$ However, week-long cultures of spheroids were possible, and reasonable cell viability could be obtained. The viability of spheroids on the HGC surface was higher than that on the GC surface. We investigated whether the spheroids could maintain prolonged spontaneous contractile activity. A 10-day follow-up analysis revealed that the contractile ability of the spheroids on the GC surface was diminished. Surprisingly, in the case of the HGC surface, the contractile ability of cardiomyocytes was retained (Supplementary Movie S4). In addition, the spheroids expressed the cardiomyocyte-specific marker cardiac troponin I (cTnI) and the gap junction-specific marker connexin (Cx43) (Figure 3f). Immunohistochemical analysis revealed robust expression of cTnI and Cx43 in spheroids on the HGC surface. In contrast, the expression of cTnI and Cx43 in spheroids on uncoated and GC-coated dishes was far less prominent. These results indicate that the HGC hydrogel is suitable for $3 \mathrm{D}$ cell cultures and does not promote any phenotypic changes.

\section{Formation of ADSC/cardiomyocyte co-culture spheroids}

Finally, we evaluated the ability of the HGC-coated plates to form co-culture spheroids with concentric layers (Figure 4). Methods for the generation of co-culture spheroids using hanging drop plates have been widely studied to produce a more biomimetic microenvironment. Although these efforts have improved the quality of spheroid manipulation, the procedures are still complex and timeconsuming, and they require non-standard equipment. ${ }^{6,18,19}$ In addition, these methods involve laborious pipetting steps to generate spheroids, rendering the scale-up process difficult. ${ }^{6}$ To overcome these limitations, we suggest a new co-culture spheroid formation method 
a

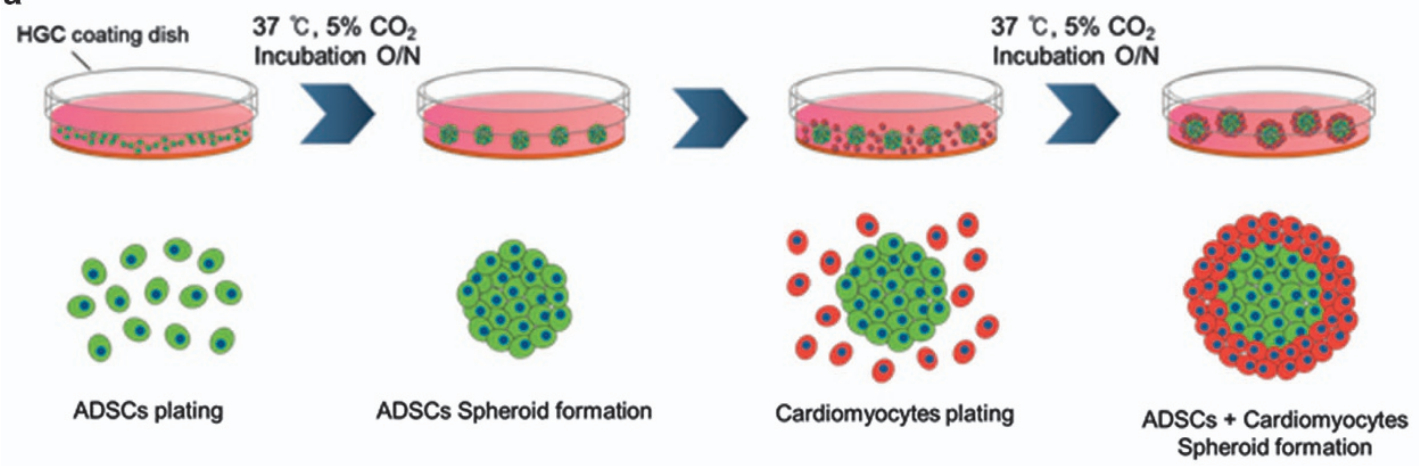

b

DAPI / ADSCs / Cardiomyocytes

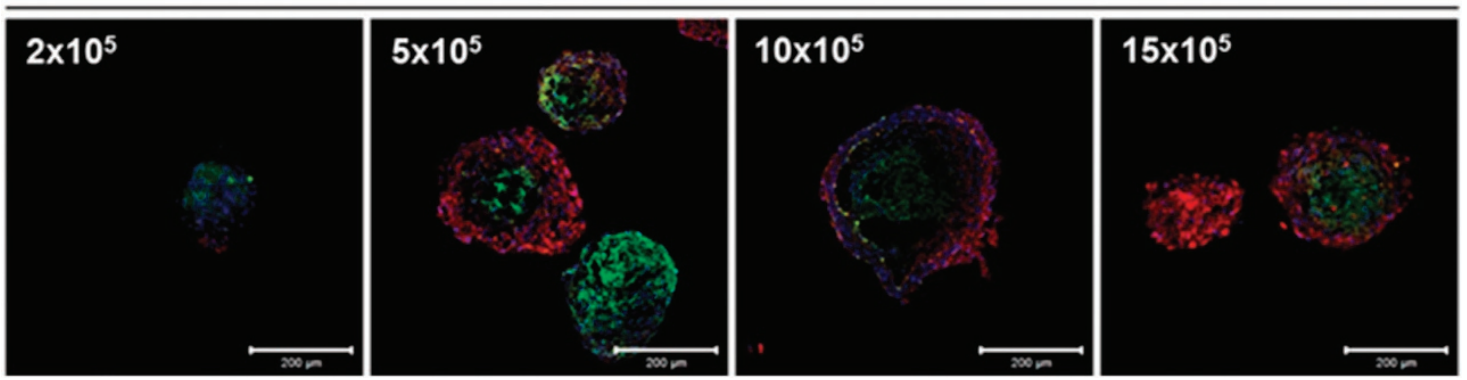

Figure 4 (a) Schematic depiction of the formation of ADSC/cardiomyocyte co-culture spheroids on HGC-coated plate. (b) Confocal fluorescence microscopy images of ADSC/cardiomyocytes co-cultured spheroids according to the number of cardiomyocytes. The spheroids were stained for ADSCs (green), cardiomyocytes (red) and cell nuclei (blue).

that uses a thermo-reversible HGC hydrogel and is applicable to various types of cells for spheroid formation (Figure 4a). This method greatly simplifies the multiple pipetting steps that are required for the formation of co-culture spheroids. Figure $4 \mathrm{~b}$ shows images of the $3 \mathrm{D}$ concentric layer patterning of CellTracker green-labeled ADSCs and red-labeled cardiomyocytes within a spheroid. The number of ADSCs was fixed at a cell density of $5 \times 10^{5}$ cells per well. On day 1 , ADSCs were observed to form spheroids with an average size of $130 \mu \mathrm{m}$. After the formation of ADSC spheroids, cardiomyocytes were added. On day 2, the cardiomyocytes (red cells) began to attach to the outer periphery of the ADSC spheroids (green cells) and subsequently merged into a single spheroid with ADSCs (green cells). Spheroids with concentric layers formed at a cell density ratio of 1:1 (green:red cells), whereas spheroids without concentric layers (only red spheroids) formed at a cell density ratio of 1:3 (green:red cells). The advantage of co-culturing is that the cell-cell interactions of natural tissues can be reproduced, a process that cannot be achieved by using synthetic factors. ${ }^{20}$ When two types of cells were plated sequentially on the HGC hydrogel, the spheroids that formed from the initially plated cells were spontaneously surrounded by the subsequently plated cells during culturing. Spheroids of this type may be directly affected by cytokines from the co-cultured cells. This $3 \mathrm{D}$ co-cultured spheroid system can be applied to tissue engineering processes for the formation of artificial tissues or organs and the replacement of failed regions, or as a model for the screening of drug toxicity and efficacy. ${ }^{21}$

\section{CONCLUSION}

In summary, we developed GC-based thermo-reversible hydrogels that can provide a facile, convenient and reproducible method for the formation of cell spheroids. The spontaneous formation of spheroids on HGC-coated plates was completed within 1 day, and the size of the spheroids varied with the cell density. Furthermore, this system can be useful for co-culturing heterotypic cells to form spheroids that mimic biological systems. The preparation of thermo-reversible hydrogels does not require any additional processes, such as the use of chemical crosslinkers for hydrogel formation or trypsin-EDTA treatment for cell retrieval, which may induce cell injury and damage. Most importantly, our hydrogel-based homo-/heterotype spheroid culture system does not require any complicated strategies such as micro-patterning. Compared with other conventional spheroid culture methods, this system can readily form a large number of spheroids within a short time and maintain their cellular function over a long period. Therefore, we conclude that this technique will be highly useful for tissue engineering, artificial organ development, drug screening models and cell production scale-up.

\section{ACKNOWLEDGEMENTS}

This study was supported by an INNOPOLIS Foundation grant funded by the Korean government (Ministry of Science, ICT \& Future Planning) through WINOVA (grant number 2014DD023), the Development of High Medical Technology Project (HI14C2755) of KHIDI, Korea, the Cooperative Research Program for Agriculture Science and Technology Development (Project No. PJ009956), Rural Development Administration, Republic of Korea and National Research Foundation of korea grant funded by Korean Government (NRF-2016M3A9B4919616). This study was also partially supported by research funds awarded by Chungnam National University in 2016. We thank Eun Hee Han of the Korea Basic Science Institute (Daejeon, Republic of Korea) for her technical support in the confocal microscopy analysis. 
1 Edmondson, R., Broglie, J. J., Adcock, A. F. \& Yang, L. Three-dimensional cell culture systems and their applications in drug discovery and cell-based biosensors. Assay Drug Dev. Technol. 12, 207-218 (2014).

2 Tibbitt, M. W. \& Anseth, K. S. Hydrogels as extracellular matrix mimics for 3D cell culture. Biotechnol. Bioeng. 103, 655-663 (2009).

3 Lee, J., Cuddihy, M. J. \& Kotov, N. A. Three-dimensional cell culture matrices: state of the art. Tissue Eng. Part B Rev. 14, 61-86 (2008).

4 Justice, B. A., Badr, N. A. \& Felder, R. A. 3D cell culture opens new dimensions in cellbased assays. Drug Discov. Today 14, 102-107 (2009).

5 Reininger-Mack, A., Thielecke, H. \& Robitzki, A. A. 3D-biohybrid systems: applications in drug screening. Trends Biotechnol. 20, 56-61 (2002).

6 Hsiao, A. Y., Tung, Y. C., Qu, X., Patel, L. R., Pienta, K. J. \& Takayama, S. 384 hanging drop arrays give excellent Z-factors and allow versatile formation of co-culture spheroids. Biotechnol. Bioeng. 109, 1293-1304 (2012).

7 Huang, Y.,C., Chan, C. C., Lin, W. T., Chiu, H. Y., Tsai, R. Y., Tsai, T. H., Chan, J. Y. \& Lin, S. J. Scalable production of controllable dermal papilla spheroids on PVA surfaces and the effects of spheroid size on hair follicle regeneration. Biomaterials 34 , 442-451 (2013).

8 Young, T. H., Lee, C. Y., Chiu, H. C., Hsu, C. J. \& Lin, S. J. Self-assembly of dermal papilla cells into inductive spheroidal microtissues on poly(ethylene-co-vinyl alcohol) membranes for hair follicle regeneration. Biomaterials 29, 3521-3530 (2008).

9 Acikgoz, A., Giri, S., Cho, M. G. \& Bader, A. Morphological and functional analysis of hepatocyte spheroids generated on poly-HEMA-treated surfaces under the influence of fetal calf serum and nonparenchymal cells. Biomolecules 3, 242-269 (2013).

10 Yeh, H. Y., Liu, B. H., Sieber, M. \& Hsu, S. H. Substrate-dependent gene regulation of self-assembled human MSC spheroids on chitosan membranes. BMC Genomics 15, 10 (2014).

11 Liu, B. H., Yeh, H. Y., Lin, Y. C., Wang, M. H., Chen, D. C., Lee, B. H. \& Hsu, S. H. Spheroid formation and enhanced cardiomyogenic potential of adipose-derived stem cells grown on chitosan. Biores. Open Access 2, 28-39 (2013).

12 Yeh, H. Y., Liu, B. H. \& Hsu, S. H. The calcium-dependent regulation of spheroid formation and cardiomyogenic differentiation for MSCs on chitosan membranes. Biomaterials 33, 8943-8954 (2012).

13 Le Tien, C., Lacroix, M., Ispas-Szabo, P. \& Mateescu, M. A. N-acylated chitosan: hydrophobic matrices for controlled drug release. J Control. Release 93, 1-13 (2003).

14 Jeong, B. \& Gutowska, A. Lessons from nature: stimuli-responsive polymers and their biomedical applications. Trends Biotechnol. 20, 305-311 (2002).
15 Lee, T. T., García, J. R., Paez, J. I., Singh, A., Phelps, E. A., Weis, S., Shafiq, Z., Shekaran, A., del Campo., A. \& García, A. J. Light-triggered in vivo activation of adhesive peptides regulates cell adhesion, inflammation and vascularization of biomaterials. Nat. Mater. 14, 352-360 (2015).

16 Cheng, M., Deng, J., Yang, F., Gong, Y., Zhao, N. \& Zhang, X. Study on physical properties and nerve cell affinity of composite films from chitosan and gelatin solutions. Biomaterials 24, 2871-2880 (2003)

17 Anada, T., Fukuda, J., Sai, Y. \& Suzuki, O. An oxygen-permeable spheroid culture system for the prevention of central hypoxia and necrosis of spheroids. Biomaterials 33 , 8430-8441 (2012).

18 Cavnar, S. P., Salomonsson, E., Luker, K. E., Luker, G. D. \& Takayama, S. Transfer, imaging, and analysis plate for facile handling of 384 hanging drop 3D tissue spheroids. J. Lab. Autom. 19, 208-214 (2014).

19 Tung, Y. C., Hsiao, A. Y., Allen, S. G., Torisawa, Y. S., Ho, M. \& Takayama, S. High-throughput 3D spheroid culture and drug testing using a 384 hanging drop array. Analyst 136, 473-478 (2011).

20 Paschos, N. K., Brown, W. E., Eswaramoorthy, R., Hu, J. C. \& Athanasiou, K. A. Advances in tissue engineering through stem cell-based co-culture. J. Tissue Eng. Regen. Med. 9, 488-503 (2015).

21 Ramaiahgari, S. C., den Braver, M. W., Herpers, B., Terpstra, V., Commandeur, J. N., van de Water, B. \& Price, L. S. A 3D in vitro model of differentiated HepG2 cell spheroids with improved liver-like properties for repeated dose high-throughput toxicity studies. Arch Toxicol. 88, 1083-1095 (2014).

(c) (i) This work is licensed under a Creative Commons Attribution 4.0 International License. The images or other third party material in this article are included in the article's Creative Commons license, unless indicated otherwise in the credit line; if the material is not included under the Creative Commons license, users will need to obtain permission from the license holder to reproduce the material. To view a copy of this license, visit http:// creativecommons.org/licenses/by/4.0/

(C) The Author(s) 2016

Supplementary Information accompanies the paper on the NPG Asia Materials website (http://www.nature.com/am). 